\title{
L'œuvre encyclopédique de Charles-Joseph Panckoucke
}

\author{
Christophe Rey \\ Université de Picardie Jules Verne, LESCLaP (CERCLL) \\ christophe.rey@u-picardie.fr
}

\section{Introduction}

Le nom de Charles-Joseph Panckoucke est traditionnellement connu comme étant celui de l'un des tout premiers magnats de la presse française des Lumières. Éditeur de journaux très influents tels que le Moniteur universel (1789), journal devenu «le journal parlementaire le plus important de la Révolution $»^{1}$, et le Gazettin (1790), «quotidien moins influent et plus radical qui sert d'antidote à la semi-officielle Gazette de France $»^{2}$, Panckoucke reste en revanche moins connu pour son rôle déterminant dans la diffusion de l'encyclopédisme du XVIII' siècle $^{3}$.

A travers cet exposé, nous nous proposons de retracer le parcours éditorial encyclopédique de cet éditeur lillois habité toute sa vie par l'obsession encyclopédique.

Après avoir rapidement rappelé les multiples facettes éditoriales de ce dernier, nous esquisserons les traits d'une aventure encyclopédique dont le point de départ est la publication du Grand Vocabulaire François (1767-1774), une œuvre originale mais très peu décrite en raison des accusations de plagiat portées à son encontre.

Nous poursuivrons notre parcours en rappelant les rôles successifs qu'a eus Panckoucke dans la publication de l'Encyclopédie ou Dictionnaire raisonné de Diderot et d'Alembert et de ce que nous avons appelé ses « suites », à savoir 1) les volumes du Supplément (1776-1777), 2) la Table analytique (1780), et ses rééditions 3) l'Encyclopédie de Genève (1770-1776) et 4) l'Encyclopédie du Lac ou Encyclopédie Pellet (1777-1779).

Nous accorderons une place toute particulière à la présentation de l' «encyclopédie suprême » que Panckoucke avait rêvé de réaliser à partir de 1768, une encyclopédie qui a monopolisé tous ses efforts pendant de nombreuses années et l'a mené plusieurs fois au bord de la faillite : l'Encyclopédie méthodique (1782-1832).

Nous achèverons notre exposé en rappelant que Panckoucke a également participé à l'édition d'ouvrages mois connus et qui pourtant illustrent la longue passion qu'il a voué aux dictionnaires et aux encyclopédies. Nous présenterons alors succinctement le Dictionnaire universel des sciences morale, économique, politique et diplomatique; ou Bibliothèque de l'homme-d'état et du citoyen (1777-1778) et le Répertoire universel et raisonné de jurisprudence civile, criminelle, canonique et bénéficiale (17751783).

\section{Un éditeur aux facettes multiples}

Afin de très rapidement présenter ce fils d'éditeur lillois et de ne pas trop paraphraser ce qui a déjà été écrit ailleurs ${ }^{4}$, nous pouvons préciser que Panckoucke a été le libraire-éditeur officiel de l'Imprimerie Royale et de l'Académie Royale des Sciences. Il s'est également distingué comme intime des plus grands philosophes et intellectuels de son siècle. Ces relations lui ont permis de proposer de nombreuses éditions littéraires de prestige, ainsi que l'illustrent par exemple ses publications des œuvres de Voltaire ou Rousseau. Mentionnons également le rachat judicieux qu'il a fait des fonds LAMBERT et DURAND en 1762-1764, fonds contenant de nombreuses œuvres littéraires de renom. 
La facette journalistique de Panckoucke commence avec la publication de 1759 à 1761 de journaux tels que Annonces, Affiches et Avis divers pour les Pays-Bas Français. Elle se poursuit avec la publication de journaux comme le Mercure de France (1778-1792) bénéficiant d'une renommée plus grande, mais prend surtout son envol dans la période post-révolutionnaire, durant laquelle il publie le Moniteur universel (1789), la Gazette de France (1789-1792) et le Gazettin (1790-1792).

En ce qui concerne l'aventure encyclopédique de l'éditeur, celle-ci commence peut-être lorsqu'après avoir quitté la province et s'être rendu à Paris il devient secrétaire du libraire éditeur de l'Encyclopédie, Le Breton :

Une intervention amicale en haut-lieu et un pot-de-vin conséquent au libraire Le Breton suffirent pour que l'apprenti de huit jour fût reçu maître le 2 septembre 1762, désormais habilité à tenir boutique et à vendre des livres dans la capitale. (Mollier, $1998: 24)$

Alors que Jean-Yves Mollier $(1988,24)$ n'hésite pas à décrire un homme « opportuniste », ayant « flairé le 'créneau porteur' de son siècle, la publication d'ouvrages scientifiques, littéraires et philosophiques, trop délaissés encore par ses confrères ", nous préférons évoquer la passion que Panckoucke a pu vouer aux dictionnaires et surtout aux encyclopédies. Cette passion l'a d'ailleurs conduit plusieurs fois au bord de la faillite et a dévoré de nombreuses années de sa vie.

Nous nous proposons à présent de reconstituer ce parcours marqué à la fois par le sceau de la passion et par celui des guerres éditoriales.

\section{Le GVF, premier fait d'armes lexicographique de Panckoucke}

La première production lexicographique majeure de Panckoucke paraît dès 1767, soit près d'un an avant la publication des derniers volumes de texte de l'Encyclopédie ou Dictionnaire raisonné de Diderot et D'Alembert. Le Grand Vocabulaire François (désormais GVF) est un ouvrage qui par son titre et son sous-titre illustre déjà le désir d'exhaustivité poursuivi par Panckoucke :

\section{LE GRAND VOCABULAIRE FRANÇOIS CONTENANT}

$1^{\circ}$. L'explication de chaque mot considéré dans ses diverses acceptions grammaticales, figurées, synonymes et relatives.

$2^{\circ}$. Les loix de l'Orthographe; celles de la Prosodie, ou Prononciation, tant familière qu'oratoire; les Principes généraux \& particuliers de la Grammaire; les règles de la Versification, \& généralement tout ce qui a rapport à l'Eloquence \& à la Poësie.

$3^{\circ}$. La Géographie ancienne \& moderne,; le Blason, ou l'Art héraldique; la Mythologie; l'Histoire naturelle des Animaux, des Plantes \& des Minéraux; 1'Exposé des Dogmes de la Religion, \& des Faits principaux de l'Histoire Sacrée, Ecclésiastique \& Profane.

$4^{\circ}$. Des détails raisonnés \& philosophiques sur l'Economie, le Commerce, la Marine, la Politique, la Jurisprudence Civile, Canonique \& Bénéficiale; l'Anatomie, la Médecine, la Chirurgie, la Chimie, la Physique, les Mathématiques, la Musique, la Peinture, la Sculpture, la Gravure, l'Architecture, \&c. \&.

PAR UNE SOCIÉTÉ DE GENS DE LETTRES.

Nous verrons ci-dessous qu'en dépit de ce programme conséquent, le GVF n'a pas connu le succès escompté par son éditeur et qu'il a même été au cœur d'une vaste polémique scientifique.

\subsection{Les éditeurs du Grand Vocabulaire François}

Publié de 1767 à 1774, le Grand Vocabulaire François appartient à la lignée des nombreux dictionnaires ou encyclopédies parus durant l'intense période d'activité scientifique que constitue le siècle des Lumières. A l'image de plusieurs de ses prédécesseurs de renom, il possède la particularité d'avoir été 


\subsection{Les auteurs du Grand Vocabulaire François}

Si l'identité des éditeurs du GVF est tout à fait claire, celle de ses auteurs l'est en revanche beaucoup moins. Les rédacteurs scientifiques de l'ouvrage sont en effet désignés sur la page de titre de chaque volume sous l'appellation globalisante de «Société de gens de Lettres ». Mais qui sont précisément ces gens de Lettres? Les seuls noms que laissent apparaître les textes de présentation de l'ouvrage sont ceux de Joseph Nicolas Guyot, Sébastien-Roch-Nicolas de Chamfort et Ferdinand Camille Duchemin de la Chesnaye. Ces trois seuls auteurs ont-ils pu rédiger un monument de cette taille ? Nous n'en savons rien et l'absence de signature des articles rajoute de l'opacité à cette question. Précisons d'ailleurs que les bibliographies mentionnant notre œuvre - citons en exemple la bibliographie proposée par le Musée Virtuel des Dictionnaires ${ }^{6}$ - évoquent ses auteurs sous la mention « Auteurs anonymes ».

\subsection{La taille du Grand Vocabulaire François}

Conformément au gigantisme caractérisant le mouvement encyclopédique, le GVF est doté d'une taille importante. En effet, pas moins de 30 volumes au format in-quarto le composent, ce qui représente, d'après le fac-similé qu'en ont donné les éditions Slatkine reprints en $2005^{7}$, précisément 18240 pages. Un tel volume permet donc à l'ouvrage de Panckoucke de figurer parmi les œuvres de grande taille et nous laisse ainsi quelque peu circonspect sur les raisons de son faible retentissement scientifique et sur la rareté des études lui ayant été consacrées.

\subsection{Un ouvrage au cœur de la tourmente}

Un examen de l'ancrage historique et théorique du GVF permet de dresser le portrait d'un ouvrage positionné comme un héritier de l'Encyclopédie et comme un anti-Trévoux ${ }^{8}$. Néanmoins, sa proximité avec l'ouvrage de Diderot et D'Alembert et sa publication entre les deux dernières éditions du Dictionnaire universel de Trévoux (1752 et 1771) sont à l'origine des accusations de plagiat portées à son encontre. Panckoucke a d'ailleurs tenté en vain de se défendre dans l'Avertissement du tome II du GVF :

Nous avons profité, sans doute, des traits de lumières répandus dans l'Encyclopédie, nous en sommes convenus précédemment; mais une preuve évidente qu'il n'est pas possible que le grand Vocabulaire françois soit la copie de cet excellent Livre, \& que les plans de ces deux ouvrages diffèrent essentiellement l'un de l'autre, c'est que depuis le monosyllabe A, jusqu'au substantif AIGUILLE, qui commence notre second volume, nous expliquons plus de deux mille six cens mots, tandis que l'Encyclopédie n'en traite que neuf cens, encore ne les considère-t-elle pas sous toutes les acceptions dont ils sont susceptibles.

Quant au Dictionnaire de Trévoux, nous osons assurer que nous n'en avons pas même tiré une seule phrase : les erreurs nombreuses que nous en indiquons dans ce Livre, dont la nomenclature n'est d'ailleurs depuis A jusqu'à AIGUILLE, que de quatorze cens trente mots, auroient dû nous mettre à l'abri de tout soupçon de plagiat. Mais pourquoi aurions-nous copié des Dictionnaires, tandis que nous avions sous les yeux les sources où ils ont puisé, \& en général les différens écrits des meilleurs Auteurs de tous les siècles?

Il seroit inutile de nous arrêter plus long-temps sur une accusation de ce genre : elle est trop peu fondée, pour qu'elle doive intéresser nos Lecteurs; nous les prierons seulement de comparer le grand Vocabulaire françois avec les Livres dont on prétend qu'il n'est que la copie; ce moyen nous justifiera mieux que tout ce que nous pourrions dire.

Ces détails de Grammaire qui ne sont propres qu'au grand Vocabulaire françois, suffisent, sans doute, déjà pour réfuter puissamment toute imputation de ressemblance 
En dépit de ces propos de défense, les travaux de Leca-Tsiomis (2002) montrent que l'héritage encyclopédique se manifeste dans le GVF par la reprise «souvent à l'identique, des articles à contenu technique et scientifique, mais [...] aussi en matière de langue courante » du dictionnaire de Trévoux.

Parallèlement, un examen de quelques articles pris au hasard dans le GVF suffit à montrer que ces derniers constituent en effet une marqueterie le plus souvent obtenue à partir de portions de texte empruntées à l'Encyclopédie, au Dictionnaire Universel de Trévoux et même à la quatrième édition du Dictionnaire de l'Académie Française (1762) (Leca-Tsiomis, 2002).

\title{
3.5 Un ouvrage néanmoins audacieux et original
}

Bien que très proche, voire parfois trop proche de ses prédécesseurs, le GVF possède néanmoins des caractéristiques qui font de lui un ouvrage digne d'un véritable intérêt.

\subsubsection{De vrais choix lexicographiques}

L'une des caractéristiques essentielles du Grand Vocabulaire François est sans aucun doute l'ambition qui semble l'habiter. Dictionnaire censé renfermer seul « la totalité des mots de tous les Dictionnaires »", le GVF adopte des choix lexicographiques intéressants :

\begin{abstract}
Le grand Vocabulaire doit être le code le plus complet de la Langue françoise \& des Belles-Lettres : chaque mot y est expliqué dans tous les sens qui lui sont propres : on remarque les nuances qui le différencient des autres mots auxquels il peut avoir rapport. Si c'est un adjectif, on enseigne s'il doit suivre ou précéder nécessairement ou indifféremment le substantif auquel il appartient : si c'est un verbe, on assigne son régime, \& par quelles particules il doit être lié avec l'infinitif d'un autre verbe : s'il est irrégulier, on le conjugue : s'il est régulier, on indique les règles pour le conjuguer, \& quels auxiliaires forment ses temps composés, quand il est neutre. Chaque mot d'usage est d'ailleurs suivi de sa quantité prosodique, partie jusqu'à présent si négligée, \& cependant si essentielle aux graces, à la purteté \& à l'harmonie du langage; on développe les abus de l'orthographe actuelle, avec les moyens d'y remédier; en un mot, on ne laisse rien à désirer sur la manière d'écrire $\&$ de prononcer correctement. (Grand Vocabulaire François, Avertissement, Tome II : iij)
\end{abstract}

La systématisation de toutes ces informations d'ordre orthographique, prosodique et syntaxique confère au GVF une originalité véritable par rapport à tous ses prédécesseurs, une originalité qui constitue d'ailleurs l'un de ses intérêts scientifiques principaux.

\subsubsection{Un ouvrage rejetant la « philosophie ferme et hardie » de l'Encyclopédie}

En plus de proposer des choix lexicographiques particulièrement intéressants, le GVF se distingue également des autres répertoires par l'esprit philosophique qu'il véhicule. Bien qu'héritier de l'Encyclopédie de Diderot et D'Alembert, l'ouvrage de Panckoucke manifeste une certaine distance théorique majeure avec celle-ci.

En effet, alors que l'ouvrage de Diderot et d'Alembert manifeste la philosophie ferme et hardie que nous lui connaissons, le GVF apparaît comme « religieusement et politiquement aseptique $»^{10}$ :

[...] des lignes de parenté profondes existent entre l'Encyclopédie et le Trévoux: un certain souci passionné de la langue, le sens de la controverse et de la polémique, la combativité des définitions. Il y a au contraire, dans l'entreprise de Panckoucke, une 
Toujours selon Leca-Tsiomis, le conformisme définitionnel des vocabulistes, «tant idéologique que méthodologique », se vérifie d'autant plus lorsque les articles de l'Encyclopédie sont signés de la plume de Diderot. Leur homologue du GVF traduit le plus souvent un «travail d'édulcoration, sur le plan politique, religieux et philosophique $»^{11}$.

La distance prise par le Grand vocabulaire français par rapport à l'Encyclopédie traduit une réelle originalité, une originalité qui globalement se manifeste par une volonté d'expurger la lexicographie des plus grosses polémiques religieuses, morales et politiques de l'époque.

\title{
4 L'Encyclopédie de Diderot et D'Alembert et ses « suites »
}

L'un des plus importants rôles éditoriaux joués par Charles-Joseph Panckoucke concerne la célèbre Encyclopédie de Diderot et d'Alembert. Après en avoir racheté les droits, l'éditeur lillois va en effet contribuer de manière intense à la diffusion de cette encyclopédie et des différentes suites qui lui seront données.

\subsection{La réédition de Genève}

Bien qu'ayant nourri dès 1768 le projet de mettre sur pied une édition renouvelée de l'Encyclopédie, Panckoucke ne réalisera ce projet que de nombreuses années plus tard, à travers la publication de l'Encyclopédie méthodique. Il va néanmoins entre temps assurer des rééditions de cet ouvrage. La première réédition paraitra à Genève dans un format in-folio et sera assurée en association avec Gabriel Cramer. S. Tucoo-Chala détaille cette association franco-suisse :

\begin{abstract}
Dans l'acte d'association du 26 juin 1770 (dit acte de Bouillon), il est précisé que Gabriel Cramer et son associé, Samuel de Tournes, 'se chargent de faire imprimer les quatorze volumes de discours tirés à deux mille...' (art. 4). Panckoucke, de son côté, s'engageait à imprimer les huit volumes de planches après en avoir retouché les cuivres (art. 3), à fournir aux imprimeurs suisses les caractères nécessaires à l'impression des volumes de discours (art. 6) ainsi que le papier (art. 7) afin que l'édition genevoise fût en tout semblable à ce qui avait été commencé à Paris. Financièrement les charges sont supportées pour un tiers par les Suisses (art. 1), pour deux tiers par Panckoucke et ses associés parisiens (auxquels il rachète les parts avant 1775). (TUCOO-CHALA, $1977: 300$ )
\end{abstract}

Les premiers volumes de cette réédition vont paraitre dès 1768 et les derniers en 1776, soit peu de temps avant la publication du Supplément à l'Encyclopédie.

\subsection{Le Supplément}

Ayant dû se «contenter» dans un premier temps de ne donner qu'une réédition au format in-folio de l'Encyclopédie, Panckoucke a en revanche joué un rôle majeur dans la publication du Supplément à l'Encyclopédie paru sous la forme de cinq volumes de texte et un de planches de 1776 à 1777. L'éditeur lillois, ainsi que le souligne Robert Darnton ci-dessous, constitue en effet l'un des éléments essentiels du groupe d'éditeurs en charge de cette suite de l'Encyclopédie:

[...] le 12 avril 1771, Panckoucke forme une nouvelle association pour une série de suppléments destinés à corriger les erreurs et à combler les lacunes du texte original. Cette société se compose des actionnaires de l'entreprise de réimpression, exception faite des deux Genevois [Gabriel Cramer et Samuel de Tournes] et de Lambot qui avait probablement revendu ses parts à Panckoucke au début de 1771. Elle comprend 
Bien que le nom de Jean-Baptiste Robinet soit le plus souvent mentionné pour désigner le principal responsable du Supplément - notamment en raison de la triple «casquette » de ce dernier, à la fois responsable du projet, éditeur et auteur de celui-ci -, Robinet ne s'avère être qu'un actionnaire minoritaire au sein de l'association d'éditeurs :

Selon l'« acte de Bouillon », original du 12 avril 1771, les parts de l'association pour le Supplément sont divisées comme suit : six vingt-quatrièmes à Cramer et Tournes; six vingt-quatrièmes à Pierre Rousseau; trois vingt-quatrièmes à Robinet; quatre vingt-quatrièmes à Panckoucke et deux vingt-quatrièmes à Brunet. Après le désistement des Genevois, leurs parts sont divisées entre Panckoucke et Brunet. (DARNTON, $1982: 35$ )

En ayant racheté la moitié des parts des éditeurs suisses Cramer et Tournes, Panckoucke est devenu le principal actionnaire du Supplément, et c'est lui-même qui a réussi à obtenir un privilège d'impression pour l'ouvrage.

\subsection{La Table analytique}

Après la publication du Supplément, Panckoucke a ensuite assuré en 1780 celle de la Table analytique et raisonnée des matières contenues dans les XXXIII volumes in-folio du Dictionnaire des sciences, des arts et des métiers et dans son Supplément.

Nous ne savons que peu de choses de cet ouvrage composé de deux tomes, publié lui aussi à la fois à Amsterdam - par Rey - et à Paris - par Panckoucke, et dont la genèse se trouve rapidement résumée dans sa préface :

La célébrité de l'Encyclopédie dans toute l'Europe, exigeoit qu'un Ouvrage destiné à faire corps avec elle, eût le degré de perfection propre à le rendre digne d'une pareille association; il exigeoit de l'unité dans le plan \& de la liaison dans les parties; il demandoit de plus qu'une personne seule se chargeât de l'éxécution; mais où trouver un homme qui fût assez versé dans les différens genres de connoissances, \& qui pût avoir assez de patience \& de tems pour lire \& analyser, la plume à la main, trente-trois Volumes in-folio, enfin qui réunît tous les talens nécessaires pour un travail aussi pénible \& aussi difficile?

M. Mouchon, Ministre de l'Eglise Françoise à Basle, a eu le courage de l'entreprendre : ses connoissances en tout genre, \& l'application qu'il a donnée à cet Ouvrage, nous répondent du succès de son travail $\&$ de la reconnoissance du public : il y a consacré huit années entières; \& ceux qui l'examineront avec attention, seront étonnés qu'il ait pu en venir à bout dans cet espace de tems : c'est le fruit de tant de veilles que nous donnons aujourd'hui au public, sous le titre de Table analytique \& raisonnée des matières contenues dans les XXXIII Volumes in-folio de l'Encyclopédie. (Table analytique, Avertissement)

S. Tucoo-Chala désigne cette table comme « un excellent abrégé de l'Encyclopédie », devant « combler les lacunes des Suppléments et plus précisément rapprocher les articles complémentaires, rétablir les articles oubliés, faire apparaître les contradictions, rapprocher les planches et leurs explications [...] ${ }^{12}$.

Parue tout juste deux ans avant les premiers volumes de l'Encyclopédie Méthodique, la Table analytique constitue, à l'image du Supplément, une véritable étape dans la transition entre l'Encyclopédie de Diderot et d'Alembert et l'« encyclopédie suprême » que Panckoucke va bientôt mettre sur pied. 


\title{
4.4 L'Encyclopédie du Lac ou Encyclopédie Pellet
}

L'évocation de l'Encyclopédie du Lac ou Encyclopédie Pellet nous ramène quelques années avant la parution de la Table analytique et permet d'évoquer une nouvelle tentative de Panckoucke pour réaliser la version corrigée et augmentée de l'Encyclopédie qu'il envisage depuis 1768.

Publiée de 1776 à 1782, 1'Encyclopédie du Lac ne constitue en fait qu'une édition au format in- $4^{\circ}$ de la première encyclopédie, une encyclopédie dont l'origine se situe au moment où Panckoucke a déjà mis au travail ses collaborateurs, et plus particulièrement son beau-frère Suard, pour la refonte de l'ouvrage de Diderot et D'Alembert :

\begin{abstract}
Armé de colle et de ciseaux, il attaque les Encyclopédies in-folio, coupe les passages contenant des erreurs et greffe sur le texte des additifs qu'il a extraits des autres ouvrages de référence [...], incorpore les notes qu'il a préparées pour son Dictionnaire sur la langue française et les nouveaux articles qu'il reçoit de son équipe d'écrivains. ${ }^{13}$
\end{abstract}

Alors que le projet de Panckoucke prend forme, ce dernier apprend en 1776 l'existence de deux éditions concurrentes de son encyclopédie : une édition in $-8^{\circ}$ prise en charge par la Société Typographique de Lausanne et une édition in- $4^{\circ}$ dirigée par l'imprimeur lyonnais Joseph Duplain réfugié à Genève et auquel l'éditeur Pellet prête son nom.

Après avoir refusé de s'associer à la première de ces deux éditions, l'éditeur lillois élabore un stratagème consistant à lui autoriser un accès en France en échange d'une somme de 24000 livres payée sous la forme d'exemplaires de cette réédition. Panckoucke s'empresse alors de vendre ces exemplaires au rabais sur le marché parisien, dépréciant ainsi l'ouvrage et obligeant la Société Typographique de Lausanne incapable de vendre ses exemplaires plus chers que ceux de Panckoucke - à en stopper la vente en France.

En ce qui concerne la seconde édition, plus susceptible de nuire à la fois à la vente de son Encyclopédie in-folio et à l'élaboration de son encyclopédie remaniée, Panckoucke réagit en s'associant à Duplain (acte de Dijon) pour la réalisation de cette encyclopédie surnommée Encyclopédie du Lac, publiée en 32 volumes, 29 de texte et 3 de planches. Conformément à l'acte d'association passé entre Duplain et Panckoucke, cette réédition de l'Encyclopédie ne portera jamais le nom de l'éditeur lillois, et bien que lui ayant permis de préserver provisoirement ses propres projets, l'a une nouvelle fois obligé à différer la réalisation de son encyclopédie remaniée :

\begin{abstract}
Si Panckoucke assume le double projet de deux Encyclopédies - Encyclopédie Suard, Encyclopédie du Lac - il ne peut le faire que de janvier 1777 (acte de Dijon) à mars de la même année. Pendant cette période, Panckoucke et la Société Typographique de Neuchâtel offrent à M. M. Rey une participation, une fois de plus refusée, à cette Encyclopédie et le 28 mars, une troisième addition de l'acte du 3 juillet 1776 fait de Suard un associé avec participation financière à l'affaire. Mais les tractations s'arrêtent net et le projet de Panckoucke disparaît une fois de plus dans la bataille économique de la concurrence. (TUCOO-CHALA, 1977 : 315)
\end{abstract}

\section{$5 \quad$ L'Encyclopédie Méthodique}

\subsection{Présentation}

L'Encyclopédie méthodique (désormais EM) est historiquement une entreprise que Panckoucke a conduite depuis 1778 .

En 1778, Panckoucke sort tout juste des tribulations de la première réédition In-folio de l'Encyclopédie de Diderot; il est également délivré des tracas des Suppléments. Mais la Table Analytique vient d'être annoncée et surtout, l'édition de l'Encyclopédie $d u$ Lac est dans sa phase optimum. (TUCOO-CHALA, $1977: 324$ ) 
En janvier 1778, un certain Deveria fait paraître un prospectus annonçant la publication prochaine d'une " édition complète de l'Encyclopédie rangée par ordre des Matières et dans laquelle on a fondu tous les Suppléments »: l'Encyclopédie méthodique. Cette nouvelle entreprise contrarie fortement Panckoucke qui se sent ainsi dépossédé de son projet initial et voit dans cette initiative une nuisance possible pour son Encyclopédie du Lac. L'éditeur lillois applique alors sa stratégie habituelle :

La première réaction, c'est la défense et la critique; la deuxième, l'association; la troisième, la prise en charge totale de l'affaire. (TUCOO-CHALA, 1977 : 325)

Panckoucke se lance en effet d'abord dans une tentative de dévalorisation du projet en décrivant Deveria comme un simple « commis chez Madame Veuve Batuty, libraire à Paris il y a 2 ans $~^{14}{ }^{14}$ et comme un libraire inexpérimenté et peu légitime pour la prise en charge d'une telle entreprise.

Dans un second temps, Panckoucke décide, en accord avec Duplain et la Société Typographique de Neuchâtel, de s'associer au projet. Le but de cette démarche est bien entendu de mettre la main sur ce projet qu'il conçoit depuis si longtemps, mais surtout - du moins au tout début du projet - de protéger le marché d'une parution trop précoce qui aurait porté préjudice à la vente de l'Encyclopédie du Lac.

Panckoucke va par la suite davantage s'investir dans ce projet qui, étant donné les nombreuses années écoulées depuis la première encyclopédie, ne peut plus en être une réédition mais une version bien différente :

Le temps d'élaboration de cette nouvelle Encyclopédie coïncidait précisément avec celui que l'écoulement des dernières rééditions de l'Encyclopédie de Diderot exigeait. L'Encyclopédie de Panckoucke pouvait désormais succéder à l'Encyclopédie Diderot pour s'adapter aux besoins intellectuels d'une nouvelle génération [...] (TUCOOCHALA, $1977: 326$ )

Par un jeu de tractations qui le caractérisent parfaitement, Panckoucke réussira à devenir actionnaire majoritaire de ce projet et à faire de cette encyclopédie son Encyclopédie méthodique.

Publiée de 1782 à 1832 à Paris (chez Panckoucke) et à Genève (chez Plomteux), l'EM se présente comme l'« oeuvre suprême » de Panckoucke, l'œuvre récompensant ses nombreuses tentatives d'une édition mise à jour de la première encyclopédie.

L'EM est le lieu d'une mutation épistémologique importante qui se traduit par des choix lexicographiques aussi judicieux que le changement d'agencement des connaissances :

[...] le grand défaut général de l'Encyclopédie, celui qui rendait cette nouvelle édition absolument nécessaire regarde le plan même. Ce défaut est la confusion des objets résultant de la loi qu'on s'était faite mal à propos de renfermer toutes les connaissances humaines dans un seul et même dictionnaire, au lieu de donner à chaque science, à chaque art son Dictionnaire particulier. (BEAUX-ARTS, Second prospectus, lij)

L'argument essentiel avancé par l'éditeur lillois en faveur d'une organisation thématique est l'inconfortable désordre que fait régner le « vice abécédaire $»^{15}$ de l'Encyclopédie :

Dans toutes les éditions de l'Encyclopédie, publiées à ce jour, les matériaux qui la composent sont accumulés \& confondus, \& n'ont d'autre ordre que celui de l'alphabet. Les objets les plus disparates se touchent, se heurtent, \& se succèdent brusquement. Les parties de cet ensemble sont brisées \& rejettées à des distances éloignées. La chaîne en est partout interrompue : enfin il naît de ce mélange un désordre dans les choses \& dans les idées, qui égare le lecteur, \& qui ne lui laisse aucun fil pour se guider dans ce vaste labyrinthe. (BEAUX-ARTS, Prospectus Général, iij)

L'EM est donc composée - selon l'inventaire dressé par G-B Watts ${ }^{16}$ - de 39 dictionnaires de matière, le tout représentant pas moins de 210 volumes et constituant un ouvrage doté d'une véritable identité : 


\begin{abstract}
Panckoucke se glorifie d'être l'architecte d'un « édifice » qui « ressemble autant à l'ancienne Encyclopédie que le palais du Louvre à une chaumière ou Saint-Pierre de Rome à une chapelle ». (DARNTON, $1982: 357$ )
\end{abstract}

\title{
5.2 Intérêts scientifiques de l'Encyclopédie Méthodique
}

Ainsi que nous l'avons succinctement souligné plus haut, l'un des intérêts scientifiques principaux de l'EM réside dans la ré-actualisation des connaissances qu'elle fournit par rapport à l'Encyclopédie de Diderot et D'Alembert.

En plus de traduire une mutation épistémologique importante en réorganisant thématiquement les connaissances, l'ouvrage de Panckoucke symbolise en effet un autre état de la science, une évolution considérable dans bien des domaines ${ }^{17}$.

Plusieurs travaux attestent ces changements scientifiques profonds. Il a par exemple été montré ailleurs que le dictionnaire Grammaire \& Littérature (1782-1786) de l'EM constituait le lieu d'une thématisation lexicale des évolutions sur la description phonétique de la langue ${ }^{18}$. De la même façon, une étude portant sur le lexique de l'histoire naturelle - décrit dans le dictionnaire Histoire naturelle des animaux (17821789) de l'EM - a permis non seulement de mettre en évidence le renouvellement des connaissances entre les deux encyclopédies, mais a également montré l'existence de nombreuses remises en cause scientifiques de l'Encyclopédie ${ }^{19}$.

Plus globalement, nous pourrions synthétiser le décalage entre les deux encyclopédies en soulignant qu'au travail de compilation d'un De Jaucourt inépuisable dans l'Encyclopédie a succédé celui de rédacteurs uniquement en charge des articles relevant de leur(s) spécialité(s) et pour la plupart membres des plus éminentes académies scientifiques.

L'EM constitue un monument de la lexicographie française qui en plus de marquer l'aboutissement de la quête encyclopédique de Panckoucke, traduit incontestablement le passage vers un nouveau siècle.

\section{D'autres projets moins connus}

Nous souhaitons achever notre exposé en évoquant très rapidement deux projets encyclopédiques plus marginaux auxquels Panckoucke a pris part et qui contribuent également à renforcer l'image de cet homme obsédé par le monde des encyclopédies.

\subsection{Dictionnaire universel des sciences morale, économique, politique et diplomatique; ou Bibliothèque de l'homme-d'état et du citoyen}

Publié de 1777 à 1783, le Dictionnaire Universel des Sciences Morales, économiques, politiques et diplomatiques ou bibliothèque de l'homme d'Etat et du citoyen est un ouvrage dirigé par J-B. Robinet, ce même Robinet avec lequel Panckoucke avait déjà collaboré pour la publication du Supplément à l'Encyclopédie.

Doté de 30 volumes au format in $-4^{\circ}$, ce Dictionnaire universel est visiblement sorti des presses de Clément Plomteux - également partenaire de Panckoucke pour l'EM - et porte l'adresse londonienne des «Libraires associés». Sous ce nom se trouvent en fait rassemblés les éditeurs Rosset de Lyon, Van Harrevelt d'Amsterdam et Panckoucke de Paris. La souscription se fait chez Elmsly à Londres.

Panckoucke n'a visiblement assuré la publication que des trois premiers volumes de cet ouvrage et Plomteux n'a pour sa part assuré que les cinq premiers volumes.

Bien que portant sur un lexique de spécialité, cette initiative symbolise à la fois le désir de Panckoucke d'embrasser tous les projets éditoriaux d'ampleur de l'époque et d'exploiter ce créneau très lucratif que représente le commerce des encyclopédies. 


\subsection{Répertoire universel et raisonné de jurisprudence civile, criminelle, canonique et bénéficiale (1775-1783)}

Le Répertoire Universel et raisonné de jurisprudence civile, criminelle, économique et bénéficiale est un ouvrage qui symbolise une nouvelle collaboration entre Panckoucke et Pierre Guyot, ce même Guyot qui vient de participer à la première aventure encyclopédique, le GVF. Avec Guyot comme éditeur scientifique à la tête d'une équipe composée par "plusieurs jurisconsultes », Panckoucke va participer à la publication de la première édition du Répertoire Universel, une édition publiée au format in-octavo, dotée de 64 volumes et publiée de 1775 à 1783.

Cette première publication de l'ouvrage va connaître certaines difficultés avec de nombreux changements de responsables. Les quatre premiers volumes sont en effet édités uniquement par le libraire J.D Dorez. Panckoucke entre ensuite dans l'affaire et prend le relais de la publication en assurant seul la diffusion des tomes 5 à 26. Du tome 27 au tome 48, un dénommé Dupuis assure conjointement avec Panckoucke la suite de la publication du Répertoire Universel. Dupuis quitte ensuite l'aventure et laisse l'éditeur lillois assurer seul la publication des tomes 49 et 50. La fin de l'ouvrage, depuis le tome 51 jusqu'au dernier tome, verra la participation d'un nouveau libraire, un dénommé Visse, lequel prendra en charge la seconde édition, publiée de 1784 à 1785 .

Portant également sur un vocabulaire de spécialité, cet ouvrage illustre lui aussi la place majeure accordée par l'éditeur lillois au genre des dictionnaires et des encyclopédies.

\section{Conclusion}

La participation de Charles-Joseph Panckoucke au mouvement de diffusion de l'encyclopédisme des Lumières, que cela soit à travers son rôle modeste en tant que rédacteur d'articles ou de sa contribution beaucoup plus importante en tant que diffuseur et éditeur d'ouvrages encyclopédiques, a pris des formes diverses.

Véritable « obsédé du monde des encyclopédies » et homme d'affaire particulièrement « opportuniste », Panckoucke a pris part à la diffusion d'ouvrages dont le rayonnement scientifique est assez hétérogène.

Sa première expérience d'ampleur, le Grand Vocabulaire Universel, est passée à la postérité comme une œuvre ayant plagié les grands répertoires de l'époque. Sans remettre en cause ces accusations, nous restons néanmoins convaincu que ce dernier possède une véritable originalité scientifique et qu'il mérite à ce titre de faire l'objet de futures investigations.

Profitant de la mode encyclopédique du siècle des Lumières, les rééditions de l'Encyclopédie, l'Encyclopédie in-folio de Genève et l'Encyclopédie du Lac ou Encyclopédie Pellet ont constitué pour Panckoucke une formidable occasion de s'enrichir et d'exercer une mainmise sur le monde de l'édition européenne.

Les «suites» de l'ouvrage de Diderot et D'Alembert, le Supplément et la Table analytique sont aujourd'hui difficilement dissociables de ce monument et lui confèrent un aspect plus abouti et mieux achevé.

Le Dictionnaire Universel et le Répertoire Universel symbolisent quant à eux le désir d'universalité qui habite Panckoucke depuis longtemps et que l'Encyclopédie Méthodique matérialise enfin. Trop peu décrite à ce jour, cette encyclopédie « suprême » n'a sans doute pas fini de livrer tous ses secrets.

\section{Références bibliographiques}

Beauzée, N., Marmontel, J.-F., (1782-1784-1786). Encyclopédie Méthodique. Grammaire \& Littérature. Paris : Panckoucke, Liège : Plomteux. 3 vol.

Darnton, R. (1982 [1979]). L'Aventure de l'Encyclopédie. 1775-1800. Un best-seller au siècle des Lumières, Paris : Perrin, 445 p. Ill. Traduction de Marie-Alyx Revellat. Préface d'Emmanuel Le Roy Ladurie. 
Diderot, D., Alembert, J. Le Rond d'., (1751-1766). Encyclopédie, ou Dictionnaire raisonné des sciences, des arts et des métiers, par une société de gens de Lettres. Stuttgart : F. Frommann Verlag - G. Holzboog, 1990.

Diderot, D., Alembert, J. Le Rond d'. (1770-1776). Encyclopédie ou Dictionnaire raisonné des sciences, des arts et des métiers par une société des gens des lettres. Genève : Panckoucke / Cramer, 35 vol. in-folio.

Diderot, D., Alembert, J. Le Rond d'. (1777-1779). Encyclopédie ou Dictionnaire raisonné des sciences, des arts et des métiers par une société des gens des lettres. Genève : Pellet; Neuchâtel: La Société Typographique.

Dictionnaire universel des sciences morale, économique, politique et diplomatique, ou Bibliothèque de l'homme d'État et du Citoyen, 1777-1778, Jean Baptiste Robinet (éd.). Londres : Chez les Libraires associés.

Doig, K.H. (1992). L’Encyclopédie méthodique et l'organisation des connaissances. Recherches sur Diderot et sur l'Encyclopédie, 12, 59-69.

Encyclopédie méthodique ou par ordre de matières par une société de gens de lettres, de savants et d'artistes; précédée d'un Vocabulaire universel, servant de Table pour tout l'Ouvrage, ornée des Portraits de MM. Diderot et d'Alembert, premiers Editeurs de l'Encyclopédie, 1782-1832, Paris : Panckoucke, Liège : Plomteux. 210 vol.

Guyot, J.-N.; Chamfort, S.R.N.; Duchemin de la Chesnaye, F.C. (1767-1774). Le grand vocabulaire françois. Paris : Panckoucke; Amsterdam : Veuve Chatelain ( \& fils) et Marc-Michel Rey.

Guyot, J.-N. (1775-1783). Répertoire universel et raisonné de jurisprudence civile, criminelle, canonique et bénéficiale, 64 vol. Paris : Panckoucke.

Leca-tsiomis, M. (2002). L’Encyclopédie et ses premiers épigones : le Grand vocabulaire français de Panckoucke et le dernier Trévoux, in Le travail des Lumières, Hommage à G. Benrekassa sous la direction de N. JacquesLefèvre, Y. Séité et al. Paris : Champion, p.455-472.

Le Dictionnaire de l'Académie française : histoire et nuances de la langue française (1694-1935). (2000). Marsanne : Éditions Redon.

Mollier, J.-Y. (1998). L'argent et les lettres : histoire du capitalisme d'édition 1880-1920, Paris : Fayard.

Mouchon, P. (1780). Table analytique et raisonnée des matières contenues dans les XXXIII volumes in-folio du Dictionnaire des sciences, des arts et des métiers et dans son Supplément, Paris, Panckoucke / Amsterdam, MarcMichel Rey, 2 vols. Stuttgart-Bad Cannstatt : Friedrich Frommann Verlag, 1967.

Rey, C. (2004). Analyse et informatisation des articles traitant de l'étude des sons dans le dictionnaire Grammaire \& Littérature de Nicolas Beauzée et Jean-François Marmontel, issu de l'Encyclopédie Méthodique. Thèse de doctorat. Aix-en-Provence.

Rey, C. (2006). A la découverte d'un monument oublié: l'Encyclopédie Méthodique, Les Cahiers de lexicologie, 88 (1), Garnier, 67-82.

Rey, C. (à paraître). De l'Encyclopédie à la Méthodique : le lexique du voyage, à partir de quelques exemples. Lez Valenciennes, Presses Universitaires de Valenciennes.

(1776-1777). Supplément à l'Encyclopédie ou Dictionnaire raisonné des sciences, des arts et des métiers, par une société de gens de lettres ; mis en ordre \& publié par $M^{* * *}$ Diderot. Amsterdam : M. Rey.

Tucco-chala, S. (1977). Charles-Joseph Panckoucke \& la Librairie française, 1736-1798. Pau : Marrimpouey Jeune; Paris : Librairie Jean Touzot.

Watts, G.-B. (1958). The Encyclopédie méthodique. Publication of the modern language association of America, LXXIII, n. 1, 348-366.

\footnotetext{
${ }^{1}$ S. Tucco-Chala, 1977.

${ }^{2}$ S. Tucco-Chala, 1977.

${ }^{3}$ Cf. Rey, 2004.

${ }^{4}$ Cf. (Darnton, 1982; Tucco-Chala, 1977, Rey, 2006 et 2004)
} 
${ }^{5}$ Rappelons que Marc-Michel Rey est le célèbre éditeur ayant publié aux Pays-Bas le Supplément (1776-1777) à l'Encyclopédie (1751-1772) de Diderot et d'Alembert.

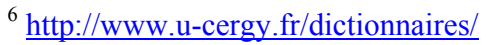

${ }^{7}$ Plus précisément, le fac-similé proposé par les éditions Slatkine reprints est celui d'une deuxième édition du Grand Vocabulaire François dont ne savons rien.

${ }^{8}$ Cf. Leca-tsiomis, 2002, « L’Encyclopédie et ses premiers épigones : le Grand vocabulaire français de Panckoucke et le dernier Trévoux ", Le travail des Lumières, Hommage à G. Benrekassa sous la direction de N. Jacques-Lefèvre, Y. Séité et al. Paris, Champion, 2002, p.455-472.

${ }^{9}$ Avertissement, tome II du GVF (1767).

${ }^{10}$ Leca-Tsiomis, 2002.

${ }^{11}$ Leca-Tsiomis, $2002: 457$.

12 Tucco-Chala, $1977: 304$.

${ }^{13}$ Darnton, $1982: 296$.

${ }^{14}$ BPN, Ms 1.233, f 54 (s.l.n.d) : "Mémoire pour les éditeurs de l'Encyclopédie de Genève portant réfutation d'un prospectus projeté par un libraire de Liège, sous le nom d'Amsterdam.".

${ }^{15}$ Doig, $1992: 60$.

${ }^{16}$ Watts, 1958.

17 Plus de soixante ans séparent les derniers volumes de textes de l'Encyclopédie des derniers volumes de l'Encyclopédie méthodique.

${ }^{18}$ Rey (2006) et Rey (2004).

${ }^{19}$ Rey, (à paraître). 